\title{
Nonlinear elastic-viscoplastic constitutive equations for aging facial tissues
}

\section{Journal Article}

Author(s):

Mazza, Edoardo; Papes, O.; Rubin, M. B.; Bodner, S. R.; Binur, N. S.

Publication date:

2005-11

Permanent link:

https://doi.org/10.3929/ethz-b-000035246

Rights / license:

In Copyright - Non-Commercial Use Permitted

Originally published in:

Biomechanics and Modeling in Mechanobiology 4(2-3), https://doi.org/10.1007/s10237-005-0074-y 


\section{Nonlinear elastic-viscoplastic constitutive equations for aging facial tissues}

Received: 17 November 2004 / Accepted: 19 April 2005/Published online: 12 August 2005

(C) Springer-Verlag Berlin Heidelberg 2005

\begin{abstract}
This paper reports on the initial stages of a project to simulate the nonlinear mechanical behavior of an aging human face. A cross-section of the facial structure is considered to consist of a multilayered composite of tissues with differing mechanical behavior. The constitutive properties of these tissues are incorporated into a finite element model of the three-dimensional facial geometry. Relatively short time (elasticviscoplastic) behavior is governed by equations previously developed which are consistent with mechanical tests. The long time response is controlled by the aging elastic components of the tissues. An aging function is introduced which, in a simplified manner, captures the observed loss of stiffness of these aging elastic components due to the history of straining as well as other physiological and environmental influences. Calculations have been performed for 30 years of exposure to gravitational forces. Progressive gravimetric soft tissue descent is simulated, which is regarded as the main indication of facial aging. Results are presented for the deformations and stress distributions in the layers of the soft tissues.
\end{abstract}

E. Mazza $(\bowtie) \cdot$ O. Papes

Institute of Mechanical Systems ETH,

8092 Zurich, Switzerland

E-mail: edoardo.mazza@imes.mavt.ethz.ch

Tel.: +41-1-6325574

Fax: + 41-1-6321145

M. B. Rubin · S. R. Bodner

Faculty of Mechanical Engineering,

Technion-Israel Institute of Technology,

32000 Haifa, Israel

N. S. Binur

The Cosmetic Surgery Center of SE Texas Port Arthur, Texas, 77642, USA

\section{Introduction}

Biomedical research is being influenced by mechanics in a number of important ways. Examples are the use of computer methods to simulate trauma (Snedeker et al. 2002), and surgical planning and surgical training using virtual reality (Brett et al. 1995; Burdea 1996; Koch et al. 1996; Avis 2000; Szekely 2003). In most cases the goal of simulations related to the face has been to produce realistic animations of facial expressions (Parke 1982; Lee et al. 1995; Koch et al. 1996; Zhang et al. 2004).

The objective of the present paper is to focus attention on the important role of realistic modeling of the mechanical response of facial tissues to loads. Previous research (Har-Shai et al. 1996, 1997) has considered mechanical experiments to study the viscoplastic properties of the skin and the underlying supportive tissue SMAS (superficial musculoaponeurotic system). Mechanical constitutive equations were developed in a simple one-dimensional form (Rubin et al. 1998) and in a more general three-dimensional form (Rubin and Bodner 2002). More specifically, the constitutive equations discussed there are in the class of elastic-viscoplastic phenomenological equations which can model the time-dependent, nonreversible material response observed in most solids. In elastic-viscoplastic theory, which is akin to nonlinear visoelasticity, the stress response is determined by history-dependent variables which are introduced through evolution equations rather than from hereditary integrals commonly used in viscoelasticity.

With regard to the modeling of facial tissues, the history-dependent variables and their associated evolution equations are introduced to model the main macroscopic response caused by the microscopic morphology of the tissues and relevant biological processes. In general, the equations and the associated material parameters are determined by comparing predictions of the theory with macroscopic experimental data. A direct connection between microscopic 
biological processes and the material parameters of this constitutive model remains illusive at this time. Nevertheless, a reasonable understanding of the mechanical behavior of the composite tissue structure should be helpful to guide new developments in clinical practice.

Regarding the aging of facial tissues, LaTrenta (2004, pp 46-47) states that: "The most commonly held theory is that facial aging is the result of progressive gravimetric soft tissue descent. Over time, the soft tissues of the face simply sag off the bones of the face, forming the distinctive wrinkles, furrows, folds, and eventual tissue redundancy of the aged face. Gravimetric soft tissue descent is complex, however, and encompasses several distinct processes. One of the most important processes is actinic damage or solar elastosis... Wrinkles become apparent in a woman's skin in her mid-30s as estrogen levels begin to decline from their peak. The dermis begins to lose collagen and elastin... Fat, unlike muscle, is supported solely by facial ligaments. After years of being pulled and stretched, these facial ligaments never regain their tautness."

In the proposed constitutive model, aging is characterized by a reduction of stiffness of facial tissues. This modeling approach is justified at the histological level by tissue degradation processes. The dermis becomes atrophied during aging, with a reduction of the volume fraction of glycosaminoglicans (specifically, the hyaluronic acid) and collagen fibers of types I, III and VII (Craven et al. 1997; Fleischmajer et al. 1972). In particular, the dermal elastic fiber network (oxitalan fibers) decreases significantly with age (Cotta-Pereira et al. 1978). In the face, these processes are accelerated by damage due to sunlight exposure (photoaging) and are complemented by solar elastosis, an accumulation of truncated, disorganized elastic fibers in the dermis (Craven et al. 1997). Progressive atrophy of superficial fat occurs in the face at distinct locations (Donofrio 2000) which might contribute to the reduction of stiffness of the SMAS (Har-Shai et al. 1998).

Age-dependent gravimetric descent is also related to the increase in volume of deep fat and the accumulation of fat around the eyes, in the cheeks and under the chin, as well as to progressive lengthening of the musculature, laxity of the ligaments and skeletal resorption (LaTrenta 2004). These processes were not considered in detail in the present model. One other typical indication of face aging that could not be simulated with the present model is the formation of deep wrinkles and skin folds. Wrinkles evolve from mimetic lines such as those around the eyes and the mouth. This process is related to localized histological modifications, in particular actinic elastosis and disappearance of oxitalan fibers (Contet-Audonneau et al. 1999), which might be influenced by the concentration of stress and strain at these locations.

In this paper, the gravimetric descent of the facial tissues is modeled by implementing the nonlinear constitutive equations into the commercial finite element computer code ABAQUS (Hibbit et al. 2002). Specifically, the constitutive equations in Rubin et al. (1998)

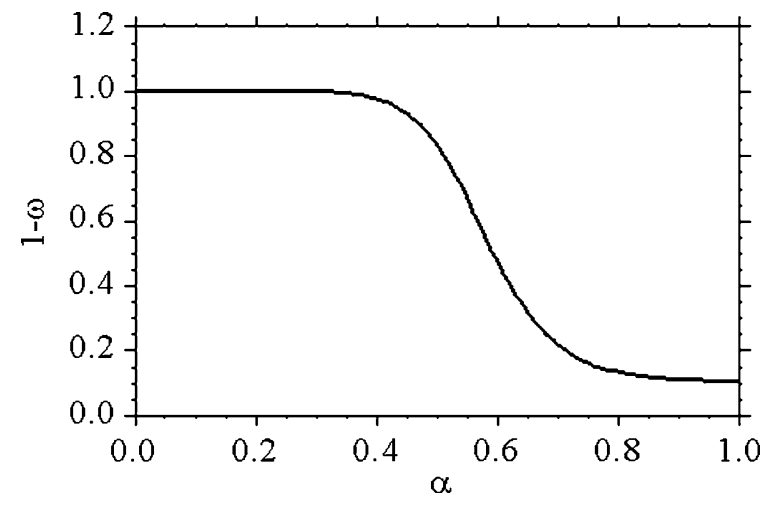

Fig. 1 Plot of the assumed stiffness reduction factor due to aging as a function of the aging parameter $\alpha$

and Rubin and Bodner (2002), which were developed for a relatively short time response to cyclic loading and relaxation tests, are generalized to include an aging function which captures the main effect of tissue degradation (loss of stiffness) exhibited in long time behavior. In particular, an aging (damage) quantity $\omega$ is proposed as a nonlinear function of an auxiliary aging parameter $\alpha$ (see Fig. 1), which itself is determined by integrating a rather simple evolution equation for its time rate of change.

The three-dimensional finite element model of the face is based on a range laser scan (Vannier et al. 1991) of a young man. Kinematic boundary conditions are defined from the description of face anatomy by Barton (2001) and LaTrenta (2004). The facial tissue is modeled as having four layers (skin, SMAS and superficial fat, deep fat and mucosa, see Fig. 2). In the present exercise, calculations have been performed for 30 years of exposure to gravitational forces from the onset of aging (age assumed to be about 30). This exposure period would correspond to about 45 years of living (assuming the face is in an erect position for $16 \mathrm{~h}$ per day). Such exposure would cause progressive gravimetric soft tissue

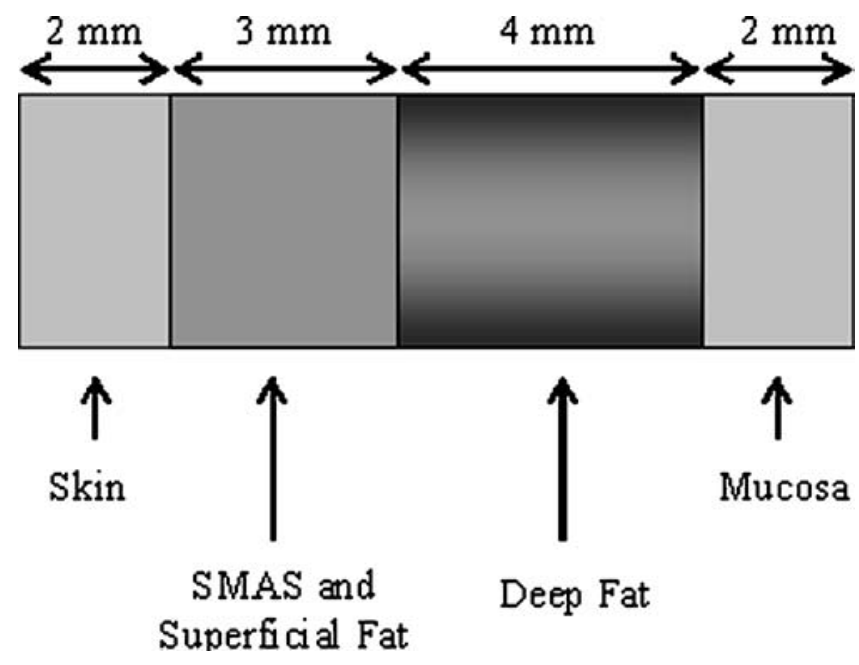

Fig. 2 Simple model of the tissue layers in the face 
descent, which is regarded as the main indication of facial aging (LaTrenta 2004, p. 46). Results are presented for the deformations and stress distributions in the layers of the soft tissues.

An outline of the paper is as follows. Section 2 describes the nonlinear constitutive equations and the aging function which are used to model each of the facial tissues. Section 3 discusses the procedure for determining the material constants so that the model matches available mechanical experimental data. Section 4 briefly discusses the numerical model of the face. Section 5 describes the main results and Sect. 6 presents conclusions and possible directions for future research.

\section{Constitutive equations}

Rubin and Bodner (2002) developed nonlinear threedimensional constitutive equations for facial tissues which are valid for arbitrarily large deformations and which produce reasonable agreement with the experimental data of Har-Shai et al. (1996). In that work, the tissue was modeled as a composite material with a fully elastic component and a dissipative component which contains both elastic and viscous elements (similar to a Maxwell model in viscoelasticity theory). The specific (per unit mass) strain energy function $\psi$ was specified in the form

$\rho_{0} \psi=\frac{\mu_{0}}{2 \mathrm{q}}[\exp (\mathrm{qg})-1]$,

where $\rho_{0}$ is the constant reference mass density, $\mu_{0}$ is a constant shear modulus and $\mathrm{q}$ controls nonlinearity. Moreover, the function $g$ was specified in an additive form

$\mathrm{g}=\mathrm{g}_{1}+\mathrm{g}_{2}+\mathrm{g}_{3}+\mathrm{g}_{4}$,

with $\left\{g_{1}, g_{2}, g_{3}\right\}$ characterizing the response of the fully elastic component and with $g_{4}$ associated with the dissipative component. Specifically, $g_{1}$ characterizes the fully elastic response to dilatation (volumetric changes), $g_{2}$ characterizes the fully elastic response to distortional deformations and $g_{3}$ characterizes the fully elastic response to extension of specific fibers. The function $g_{4}$ characterizes the response to elastic distortional deformation of the dissipative component. It is noted that the dissipative component is modeled using elastic-viscoplastic constitutive equations which for low-stress levels can produce nearly nondissipative elastic response and for high-stress levels can produce dissipative viscoplastic response. Consequently, the word "dissipative" is used to describe the dissipative component because that component has the potential to exhibit dissipation even though it may respond essentially elastically in some ranges of stress. For all stress levels, the stress tensor is a function of elastic deformation measures associated with both the fully elastic and the dissipative components.
Here, the effects of specific fibers are neglected $\left(g_{3}=0\right)$ due to lack of experimental data and the function $g_{2}$ is modified (relative to Rubin and Bodner 2002) to model the reduction in elastic stiffness caused by distortional deformations of the elastic component using an aging term $\omega$. Due to this modification, the elastic component of the present model is referred to as an "aging elastic" component rather than a "fully elastic" component. Except for this modification, most of the details of the following developments can be found in Rubin and Bodner (2002). Specifically, the functions $\left\{g_{1}\right.$, $\left.g_{2}, g_{3}, g_{4}\right\}$ are taken in the forms

$$
\begin{aligned}
& \mathrm{g}_{1}=2 \mathrm{~m}_{1}[(\mathrm{~J}-1)-1 \mathrm{n}(\mathrm{J})], \mathrm{g}_{2}=(1-\omega) \mathrm{m}_{2}\left(\beta_{1}-3\right), \\
& \mathrm{g}_{3}=0, \mathrm{~g}_{4}=\alpha_{1}-3,
\end{aligned}
$$

where $\mathbf{J}$ is the dilatation, $\beta_{1}$ and $\alpha_{1}$ are measures of the elastic distortions of the elastic and dissipative components, and $m_{1}, m_{2}$ are material constants. Details of the independent variables in the theory will be presented in Eqs. 7-19 after presenting the constitutive equations for the stresses. Next, the constitutive equation for the Cauchy stress $\mathbf{T}$ is hyperelastic with $\mathbf{T}$ obtained by a derivative of $\psi$ and $\mathbf{T}$ being separated additively into three components

$\mathbf{T}=\mathbf{T}^{(1)}+\mathbf{T}^{(2)}+\mathbf{T}^{(4)}, \mathbf{T}^{(1)}=-\mathrm{m}_{1} \mu\left[\frac{1}{\mathbf{J}}-1\right] \mathbf{I}$,

$\mathbf{T}^{(2)}=(1-\omega) \mathrm{m}_{2} \mu \mathbf{J}^{-1}\left[\mathbf{B} /-\frac{1}{3}(\mathbf{B} / \mathbf{I}) \mathbf{I}\right]$,

$\mathbf{T}^{(4)}=\mu \mathbf{J}^{-1}\left[\mathbf{B} / \mathrm{de}-\frac{1}{3}(\mathbf{B} / \mathrm{de} \cdot \mathbf{I}) \mathbf{I}\right]$,

where $\mu$ is a nonlinear shear modulus, and $\mathbf{B}^{\prime}$ and $\mathbf{B}^{\prime}{ }_{d e}$ are tensorial measures of elastic distortion of the elastic and dissipative components. In these equations, $\mathbf{T}^{(1)}$ characterizes the elastic response to dilatation, $\mathbf{T}^{(2)}$ characterizes the aging elastic response to distortional deformations, and $\mathbf{T}^{(4)}$ characterizes the dissipative response to distortional deformations. Also, $\mathbf{A} \cdot \mathbf{B}=\operatorname{tr}\left(\mathbf{A} \mathbf{B}^{\mathrm{T}}\right)$ denotes the inner product between two second-order tensors $\mathbf{A}$ and $\mathbf{B}$.

From Eq. $4 \mathrm{a}$ it can be seen that the pressure $p$ becomes

$p=-\frac{1}{3} \mathbf{T} \cdot \mathbf{I}=\mathrm{m}_{1} \mu\left[\frac{1}{\mathbf{J}}-1\right]$,

where the bulk modulus $m_{1} \mu$ is defined in terms of the nonlinear shear modulus $\mu$

$\mu=\mu_{0} \exp (\mathrm{qg})$.

The total dilation $\mathbf{J}$ is determined by the standard evolution equation

$\dot{J}=\mathbf{J D} \cdot \mathbf{I}$,

where a superposed dot denotes material time differentiation, and $\mathbf{D}$ is the symmetric part of the velocity gradient $\mathbf{L}=\cdot \partial \mathbf{v} / \cdot \partial \mathbf{x}$ 
$\mathbf{D}=\frac{1}{2}\left(\mathbf{L}+\mathbf{L}^{\mathrm{T}}\right)$.

In Eq. 4c, $\mathbf{B}^{\prime}$ is a unimodular second order tensor (Flory 1961)

$\operatorname{det}\left(\mathbf{B}^{\prime}\right)=1$,

which is a measure of total distortional deformation, and is determined by the evolution equation

$\dot{\mathbf{B}}^{\prime}=\mathbf{L B} /+\mathbf{B}^{\prime} \mathbf{L}^{\mathrm{T}}-\frac{2}{3}(\mathbf{D} \cdot \mathbf{I}) \mathbf{B}^{\prime}$.

This equation is purely kinematical and can be obtained by differentiating the expression

$\mathbf{B}^{\prime}=\mathbf{J}^{-2 / 3} \mathbf{B}$

where $\mathbf{B}$ is the left Cauchy-Green deformation tensor. Also, the scalar $\beta_{1}$ in (2.3) is a measure of total distortional deformation given by

$\beta_{1}=\mathbf{B}^{\prime} \cdot \mathbf{I}$.

In Eq. 4d, the dissipative component is modeled using equations similar to those for viscoplasticity. Specifically, $\mathbf{B}_{d e}^{\prime}$ is a unimodular second order tensor

$\operatorname{det}\left(\mathbf{B}_{\mathrm{de}}^{\prime}\right)=1$,

which is a measure of the elastic distortional deformation associated with the dissipative component, and is determined by the evolution equation

$\dot{\mathbf{B}}_{\mathrm{de}}^{\prime}=\mathbf{L B}_{\mathrm{de}}^{\prime}+\mathbf{B}_{\mathrm{de}}^{\prime} \mathbf{L}^{\mathrm{T}}-\frac{2}{3}(\mathbf{D} \cdot \mathbf{I}) \mathbf{B}_{\mathrm{de}}^{\prime}-\Gamma \mathbf{A}_{\mathrm{d}}$

Also, the scalar $\alpha_{1}$ in Eq. 3 is given by

$\alpha_{1}=\mathbf{B}_{\mathrm{de}}^{\prime} \cdot \mathbf{I}$

Equation 14 is a generalization of Eq.10 with the rate of inelastic deformation being controlled by the term $\Gamma$ Ad. In particular, when $\Gamma \mathbf{A}_{\mathrm{d}}$ vanishes, Eq. 14 has the same form as Eq. 10 and the dissipative component responds elastically. Moreover, the tensor $\mathbf{A}_{\mathrm{d}}$ controls the direction of inelastic deformation rate and is specified by the form

$\mathbf{A}_{\mathrm{d}}=\mathbf{B}_{\mathrm{de}}^{\prime}-\left\{\frac{3}{\mathbf{B}_{\mathrm{de}}^{\prime}-1 \cdot \mathbf{I}}\right\} \mathbf{I}$

which ensures that $\mathbf{B}^{\prime}{ }_{\text {de }}$ remains unimodular Eq. 13.

The scalar function $\Gamma$ in the evolution Eq. 14 requires a constitutive equation which is specified by

$\Gamma=\left[\Gamma_{1}+\Gamma_{2} \dot{\varepsilon}\right] \exp \left[-\frac{1}{2}\left\{\frac{\beta}{\beta_{\mathrm{de}}}\right\}^{2 \mathrm{n}}\right]$,

similar to that of Bodner and Partom (1975). In this equation, $\left\{\Gamma_{1}, \Gamma_{2}, \mathrm{n}\right\}$ are non-negative material constants, $\beta_{\mathrm{de}}$ is the magnitude of the deviatoric tensor $\mathbf{B}_{\mathrm{de}}^{\prime \prime}$ $\beta_{\mathrm{de}}=\sqrt{\frac{3}{2} \mathbf{B}_{\mathrm{de}}^{\prime \prime} \cdot \mathbf{B}_{\mathrm{de}}^{\prime \prime}}, \quad \mathbf{B}_{\mathrm{de}}^{\prime \prime}=\mathbf{B}_{\mathrm{de}}^{\prime}-\frac{1}{3}\left(\mathbf{B}_{\mathrm{de}}^{\prime} \cdot \mathbf{I}\right) \mathbf{I}$,

$\varepsilon$ is the equivalent total distortional strain determined by the evolution equation

$\dot{\varepsilon}=\sqrt{\frac{2}{3} \mathbf{D}^{\prime} \cdot \mathbf{D}^{\prime}}, \quad \mathbf{D}^{\prime}=\mathbf{D}-\frac{1}{3}(\mathbf{D} \cdot \mathbf{I}) \mathbf{I}$,

and $\beta$ is a measure of hardening of the dissipative component characterized by the evolution equation

$\dot{\beta}=\left[\frac{\mathrm{r}_{1} \mathrm{r}_{3}+\mathrm{r}_{2} \dot{\varepsilon}}{\mathrm{r}_{3}+\dot{\varepsilon}}\right] \Gamma \beta_{\mathrm{de}}-\mathrm{r}_{4} \beta^{\mathrm{r}_{5}}$.

with $\left\{r_{1}, r_{2}, r_{3}, r_{4}, r_{5}\right\}$ being material constants. Using the discussion in Rubin (1994), it can be shown that these constitutive equations are properly invariant under superposed rigid body motions. Also, it is noted that the term $\Gamma_{2} \dot{\varepsilon}$ in Eq. 17 was introduced in Rubin and Bodner (2002) to cause the constitutive response to be consistent with the observations on soft tissues which indicate that hysteresis loops to the same stress levels are nearly independent of strain rate over a wide range of rates (Fung 1993, p. 281).

For small values of $\beta_{\text {de }}$ relative to $\beta$, it follows from Eq. 17 that the exponential term causes the rate of inelastic deformation to be vanishingly small so the dissipative component responds essentially elastically. On the other hand, when $\beta_{\mathrm{de}}$ attains values on the order of $\beta$, then the magnitude of the inelastic response is controlled by the constants $\left\{\Gamma_{1}, \Gamma_{2}\right\}$. The sharpness of the transition between elastic and dissipative response is controlled by the constant $n$.

The evolution Eq. 20 for the hardening parameter $\beta$ models two main effects. The first term on the righthand-side of Eq. 20 causes an increase in $\beta$ due to inelastic deformation rate. The second term on the righthand-side of Eq. 20 causes $\beta$ to recover to the value zero. This overall response attempts to model effects of fluid flow in the tissue, the fluid being expelled when the tissue is deformed (hardening) and the fluid being imbibed by the tissue over time as the tissue returns to its nearly unstressed state (recovery of hardening). Specifically, the constant $r_{1}$ controls the rate of hardening during relaxation tests $(\dot{\varepsilon}=0)$, the constant $\mathrm{r}_{2}$ controls the rate of hardening during loading (large values of $\dot{\varepsilon}$ ), and the constant $\mathrm{r}_{3}$ controls the value of strain rate $\dot{\varepsilon}$ associated with the transition between these two responses. In this regard, it should be emphasized that this particular functional form for hardening is quite simplistic and can be modified when additional experimental data is available. Also, the constants $r_{4}, r_{5}$ control the rate and shape of recovery of hardening.

The functional dependence on the total strain rate $\dot{\varepsilon}$ in Eq. 17 and Eq. 20 attempts to capture differences in the observed responses to loading and relaxation tests. Specifically, for loading with large values of $\dot{\varepsilon}$ (i.e. $\Gamma_{2} \dot{\varepsilon} \gg \Gamma_{1}$ and $\left.\dot{\varepsilon} \gg \mathrm{r}_{3}\right)$ the evolution Eq.14 characterizes nearly rate-independent response. On the other hand, 
during relaxation tests (with $\dot{\varepsilon}=0$ ) the evolution Eq.14 characterizes viscoplastic rate-dependent response. Moreover, the transitions between these two types of response depend mainly on the constants $\left\{\Gamma_{2}, r_{3}\right\}$.

The main modification in Eq. 3, relative to the constitutive equations of Rubin and Bodner (2002), is the presence of the aging (damage) term $\omega$ which causes the stiffness of the elastic response to distortional deformation (see $\mathrm{g}_{2}$ in Eq. 3) to decrease with increasing $\omega$. Consistent with information in the medical literature, damage due to aging of the tissues is considered to be nonreversible. Most models for damage of materials propose an evolution equation for the time rate of change of a damage variable like $\omega$ which is a highly nonlinear function of $\omega$ and stress. Here, an alternative procedure is proposed which improves the stability of the numerical integration of the aging model. Specifically, the aging quantity $\omega$ is taken to be a function of an auxiliary aging parameter $\alpha$ which produces a generic S-like curve (see Fig. 1)

$$
\begin{gathered}
\omega=\omega(\alpha)=\left(1-\mathrm{a}_{1}\right)\left[\frac{\mathrm{a}_{2}(3 \alpha / 2)^{\mathrm{a}_{3}}}{1-\mathrm{a}_{2}+\mathrm{a}_{2}(3 \alpha / 2)^{\mathrm{a}_{3}}}\right], \\
0 \leq \mathrm{a}_{1} \leq 1,0 \leq \mathrm{a}_{2} \leq 1, \mathrm{a}_{3} \geq 0,
\end{gathered}
$$

where $\left\{a_{1}, a_{2}, a_{3}\right\}$ are material constants and $\alpha$ is determined by an evolution equation. This functional form has been chosen so that $\omega$ is bounded by zero and the value $\left(1-\mathrm{a}_{1}\right)$

$0 \leq \omega \leq 1-\mathrm{a}_{1}, \omega(0)=0, \omega(\infty)=1-\mathrm{a}_{1}$.

Also, it can be shown that

$$
\frac{\mathrm{d} \omega}{\mathrm{d} \alpha}=\frac{3\left(1-\mathrm{a}_{1}\right) \mathrm{a}_{2} \mathrm{a}_{3}(3 \alpha / 2)^{\mathrm{a}_{3}-1}}{2\left[1-\mathrm{a}_{2}+\mathrm{a}_{2}(3 \alpha / 2)^{\mathrm{a}_{3}}\right]^{2}} 0,
$$

which indicates that $\omega$ is a monotonically increasing function of $\alpha$. Next, the aging parameter $\alpha$ is determined by the relatively simple evolution equation

$\dot{\alpha}=\Gamma_{3} \varepsilon+\Gamma_{4}$,

where $\left\{\Gamma_{3}, \Gamma_{4}\right\}$ are additional material constants, with $\Gamma_{3}$ controlling the dependence on strain and $\Gamma_{4}$ controlling the dependence on other physiological and environmental effects of aging. Also, the factor $3 / 2$ in Eq. 21 was chosen for convenience and could be adjusted by changing the values of $\left\{\Gamma_{3}, \Gamma_{4}\right\}$.

With regard to more standard formulations of damage (e.g. Bodner and Chan 1986) it is noted that Eqs. 21, 23 and 24 could be combined to obtain an evolution equation directly for the damage parameter $\omega$ which is independent of the auxiliary parameter $\alpha$. However, the resulting equation would be a highly nonlinear function of $\omega$ which could cause difficulties in numerical integration. In contrast, the procedure used here embeds most of the nonlinearity in the functional form Eq. 21 for $\omega(\alpha)$ and leaves a rather simple evolution equation Eq. 24 for $\alpha$. Thus, this procedure of specifying $\omega$ as a function of an auxiliary parameter $\alpha$ may be useful for other constitutive equations that include continuum damage parameters.

Next, using the above constitutive equations and the conservation of mass

$\rho \mathbf{J}=\rho_{0}$,

which gives an expression for the current mass density $\rho$, it can be shown that the rate of material dissipation $D$

$\mathbf{D}=\mathbf{T} \cdot \mathbf{D}-\rho \dot{\psi} \geq 0$,

requires

$$
\begin{aligned}
\mathbf{D} & =\mathbf{T} \cdot \mathbf{D}-\rho \dot{\psi} \\
& =\frac{1}{2} \mu \mathbf{J}^{-1}\left[\Gamma \mathbf{A}_{\mathbf{d}} \cdot \mathbf{I}+\mathrm{m}_{2}\left(\beta_{1}-3\right) \dot{\omega}\right] \geq 0,
\end{aligned}
$$

which is satisfied for all processes since $\left\{\mu_{0}, \mathrm{~m}_{2}, \Gamma\right\}$ are nonnegative.

The material constants $\left\{\mu_{0}, \mathrm{q}, \mathrm{m}_{1}, \mathrm{~m}_{2}\right\}$ control the elastic response of the tissue. More specifically, q controls nonlinear elastic effects through the strength of the exponential function and it has insignificant influence for small deformations (with $\mu \approx \mu_{0}$ ). Consequently, the remaining elastic constants can be identified by considering small deformations. The constant $\mu_{0}$ controls the elastic shear modulus of the dissipative component and the elastic moduli of the other components have been normalized by $\mu_{0}$. Thus, the small deformation bulk modulus $\mathrm{k}_{1}$ of the tissue is given by

$\mathrm{k}_{1}=\mathrm{m}_{1} \mu_{0}$.

The small deformation shear modulus of the aging elastic component is given by $(1-\omega) \mathrm{m}_{2} \mu_{0}$. Thus, the aging function $\omega$ tends to reduce the magnitude of this shear modulus as $\omega$ and $\alpha$ increase (see Fig. 1). The material constants $\left\{a_{1}, a_{2}, a_{3}\right\}$ control the shape of the aging function Eq. 21. From Eq. 22 and Eq. 23 it is clear that $\omega$ smoothly changes from the value 0 to its maximum value $1-a_{1}$ as the aging parameter $\alpha$ increases. This means that the small deformation shear modulus of the fully aged elastic component becomes $\mathrm{a}_{1} \mathrm{~m}_{2} \mu_{0}$, which is reduced from the young tissue value of $\mathrm{m}_{2} \mu_{0}$ by the constant $\mathrm{a}_{1}$. The constant $\mathrm{a}_{2}$ controls the extent of this transition when $\alpha=2 / 3$, since

$\omega=\left(1-a_{1}\right) a_{2}$ for $\alpha=\frac{2}{3}$.

Also, the transition becomes sharper as the value of $a_{3}$ is increased. As noted earlier, the value of $\alpha=2 / 3$ in Eq. 21 was chosen for convenience but could be adjusted by changing the factor $3 / 2$ in Eq. 21 . Furthermore, the evolution Eq. 24 for the aging parameter $\alpha$ models the effect of straining on the inhomogeneous evolution of aging by the constant $\Gamma_{3}$. It is well known that exposure to sun causes aging of skin but quantifying the cumulative effect of a time-dependent history of sun exposure 
is difficult. In principle, within the context of the proposed model, the parameter $\Gamma_{4}$ could be a specified function of time to model time-dependent, but spatially uniform, effects of aging.

Of course, initial conditions must be specified for all quantities determined by evolution equations. For the calculations considered here, it is assumed that the material is initially in a stress-free state and these initial conditions are specified by

$\mathbf{J}=1, \mathbf{B}^{\prime}=\mathbf{I}, \mathbf{B}_{\mathrm{de}}^{\prime}=\mathbf{I}, \varepsilon=0, \beta=0, \alpha=0$ for $t=0$.

\section{Determination of the material constants}

The constitutive equations presented in the previous section attempt to model a number of mechanical and physiological processes. It was shown by Rubin et al. (1998) that the experimental data of Har-Shai et al. (1996) for cyclic loading and relaxation tests on relatively fresh excised samples of SMAS and skin can be modeled using elastic-viscoplastic constitutive equations without a fully elastic component for distortional deformations. However, as indicated by Rubin and Bodner (2002), the presence of such a fully elastic component is required to cause these tissues to return to their unstressed shapes when unloaded for sufficient time. This means that the relatively short time response to distortional deformations is dominated by the elasticviscoplastic component while the long time relaxation response is dominated by the aging elastic component characterized by time-dependent stiffness reduction.

Although the experiments (Har-Shai et al. 1996) revealed many important aspects of the inelastic response of these tissues, they were limited to uniaxial tension tests and they cannot provide sufficient data to determine all the material constants. Moreover, unlike metals, the response of biological tissues does not separate into nearly independent regions dominated by either elastic or viscoplastic responses. Typically, the response of these biological tissues involves regions which are influenced by both fully elastic and elastic-viscoplastic material constants simultaneously. This means that it is not possible to determine unique values for a full set of material constants with limited experimental data.

The actual facial tissues are complicated layered composite structures of epithelium, connective tissues, muscle tissues, and fat. In the simulations described in the next section, the face is modeled using four layers of tissues (see Fig. 2). Typically, surgical procedures for separating SMAS from skin cause the SMAS strip to include portions of the surrounding fat layers. Therefore, the SMAS layer identified in Fig. 2 is denoted as SMAS and superficial fat. Under the SMAS is a layer of deep fat followed by an additional layer of mucosa. It is noted that the thicknesses indicated in Fig. 2 are approximate.
The material constants are separated into three sets. One set of seven constants (Table 1)

$\left\{\mathrm{k}_{1}, \mathrm{~m}_{2}\right\},\left\{\Gamma_{3}, \Gamma_{4}\right\},\left\{\mathrm{r}_{3}, \mathrm{r}_{4}, \mathrm{r}_{5}\right\}$,

are specified here to exhibit physically reasonable response characteristics of the model where experimental data is not available, one set of three constants (Table 2)

$\left\{\mathrm{a}_{1}, \mathrm{a}_{2}, \mathrm{a}_{3}\right\}$,

which characterize the aging function, and one set of seven constants (Table 3)

$\left\{\mu_{0}, \mathrm{q}\right\},\left\{\Gamma_{1}, \Gamma_{2}, \mathrm{n}\right\},\left\{\mathrm{r}_{1}, \mathrm{r}_{2}\right\}$,

which have been determined by the available experimental data (Har-Shai et al. 1996).

More specifically, it is assumed that under load the deep fat will continue to flow and so the effect of distortional deformation on the elastic component was eliminated by taking $\mathrm{m}_{2}=0$ for this tissue. Since the deep fat does not contain the septa, which are present in the superficial fat, it is reasonable to expect that the deep fat will be weaker than the SMAS and superficial fat. However, due to lack of experimental data, the other material constants Eq. 31 not including $\mathrm{m}_{2}$, and Eq. 33

Table 1 Material constants set to exhibit specific response characteristics of the model for SMAS and superficial fat, and skin

\begin{tabular}{ll}
\hline & SMAS and superficial fat, and skin \\
\hline$k_{1}(\mathrm{GPa})$ & 2.2 \\
$\mathrm{~m}_{2}$ & $8.0 \mathrm{E}-4$ \\
$\Gamma_{3}\left(s^{-1}\right)$ & $0.8 \mathrm{E}-8$ \\
$\Gamma_{4}\left(s^{-1}\right)$ & $0.1 \mathrm{E}-9$ \\
$\mathrm{r}_{3}$ & $1.0 \mathrm{E}-8$ \\
$\mathrm{r}_{4}$ & $1.0 \mathrm{E}-4$ \\
$\mathrm{r}_{5}$ & 1.0 \\
\hline
\end{tabular}

Table 2 Material constants for the aging function for both SMAS and superficial fat, and skin

SMAS and superficial fat, and skin

\begin{tabular}{ll}
\hline $\mathrm{a}_{1}$ & 0.1 \\
$\mathrm{a}_{2}$ & 0.8 \\
$\mathrm{a}_{3}$ & $1.0 \mathrm{E}+1$ \\
\hline
\end{tabular}

Table 3 Material constants determined by available experimental data for SMAS and superficial fat, and skin

\begin{tabular}{lll}
\hline & SMAS and superficial fat & skin \\
\hline$\mu_{0}(\mathrm{MPa})$ & 3.7 & 1.7 \\
$\mathrm{q}$ & $2.5 \mathrm{E}+1$ & $3.6 \mathrm{E}+1$ \\
$\Gamma_{1}\left(s^{-1}\right)$ & $5.0 \mathrm{E}-3$ & $5.0 \mathrm{E}-3$ \\
$\Gamma_{2}\left(s^{-1}\right)$ & $2.0 \mathrm{E}+1$ & $1.0 \mathrm{E}+1$ \\
$\mathrm{n}$ & 1.0 & 0.5 \\
$\mathrm{r}_{1}$ & 0.2 & $2.0 \mathrm{E}+1$ \\
$\mathrm{r}_{2}$ & 1.3 & 2.5 \\
\hline
\end{tabular}


of the deep fat were taken to be the same as those of SMAS and superficial fat. In the absence of experimental data, the material constants of the mucosa were taken to be the same as those of skin.

Here, it is assumed that the volumetric response of each tissue is close to that of water so that the constant $\mathrm{m}_{1}$ is specified by Eq. 28 with the bulk modulus $k_{1}$ of water given in Table 1. For the fully aged tissue the stiffness to distortional deformation of the aging elastic component is controlled by the value $\mathrm{a}_{1} \mathrm{~m}_{2}$. The experimental data in Har-Shai et al. (1996) is for tissues that were excised from older patients undergoing plastic surgery and which are assumed for the present purposes to be fully aged. No experimental data for corresponding young tissue is available, so the effect of aging on the small deformation shear modulus $\mathrm{m}_{2} \mu_{0}$ of the elastic component for young tissue is speculated to be ten times its value $a_{1} m_{2} \mu_{0}$ for fully aged tissue. This assumption determines the value of $a_{1}$ in Table 2 . Figure 1 plots the stiffness reduction factor $(1-\omega)$ due to aging using the values of the constants $\left\{a_{1}, a_{2}, a_{3}\right\}$ in Table 2 . Assuming that the starting point of the simulation is around 30 years of age, taking $\alpha=1$ to correspond to 30 years of exposure to gravity and other aging effects, and assuming that the face is in an erect position for $16 \mathrm{~h}$ per day, the period $0 \leq \alpha \leq 1$ corresponds approximately to ages $30-75$.

Once the value of $a_{1}$ is set, the value of $m_{2}$ controls the shear modulus of the aging elastic component due to distortional deformation. This value was specified by simulating the response of the face (described in detail in the next section) to gravity assuming that all tissues are fully hyperelastic (i.e., ignoring the dissipative compo- nents in each tissue). The value of $\mathrm{m}_{2}$ in Table 1 was specified to produce a reasonable value for the gravimetric descent of a point in the jowl region of the face.

The constants $\left\{\mathrm{q}, \Gamma_{2}, \mathrm{n}, \mathrm{r}_{4}, \mathrm{r}_{5}\right\}$ were specified to have the same values as those given by Rubin and Bodner (2002). Then, the remaining constants $\left\{\mu_{0}, \Gamma_{1}, r_{1}, r_{2}\right\}$ in Eq. 3 were determined by matching the experimental data of Har-Shai et al. (1996), using the procedure discussed by Rubin and Bodner (2002). Specifically, the response to uniaxial stress was simulated with $\Pi_{11}$ being the engineering stress (force per unit reference area; first Piola-Kirchhoff stress) and $\varepsilon_{11}$ being the engineering strain (change in length per unit reference length). For the simulations in Figs. 3 and 4 the full nonlinear equations have been solved and the tissues have been taken to be fully aged so that $\omega$ was set to the value $\left(1-a_{1}\right)$. Also, the strain rates associated with these tests are recorded in Table 4.

Figure 3 shows that the theoretical results for fully aged tissues $\left(\omega=1-a_{1}\right)$ match the experimental data quite well for both the cyclic loading and relaxation tests of Har-Shai et al. (1996). The results in Fig. 4 compare simulations of these tests for fully aged tissues $\left(\omega=1-a_{1}\right)$ with simulations of the response of young tissues $(\omega=0)$. It is interesting to note that the short-time cyclic responses of these tissues are nearly unaffected by the factor of ten difference in magnitude of the shear modulus of the elastic component due to aging. From Fig. $4 \mathrm{~d}$ it can be seen that the simulated aging process influences the relaxation of the skin to some extent. In particular, it can be seen that young skin maintains its tension more than aged skin, even in these short-time simulated relaxation tests.
Fig. 3 Comparison of the theoretical results for aged tissues with the experimental data for cyclic loading and relaxation tests. First PiolaKirchhoff (engineering) stress $\Pi_{11}$ versus engineering strain $\varepsilon_{11}$
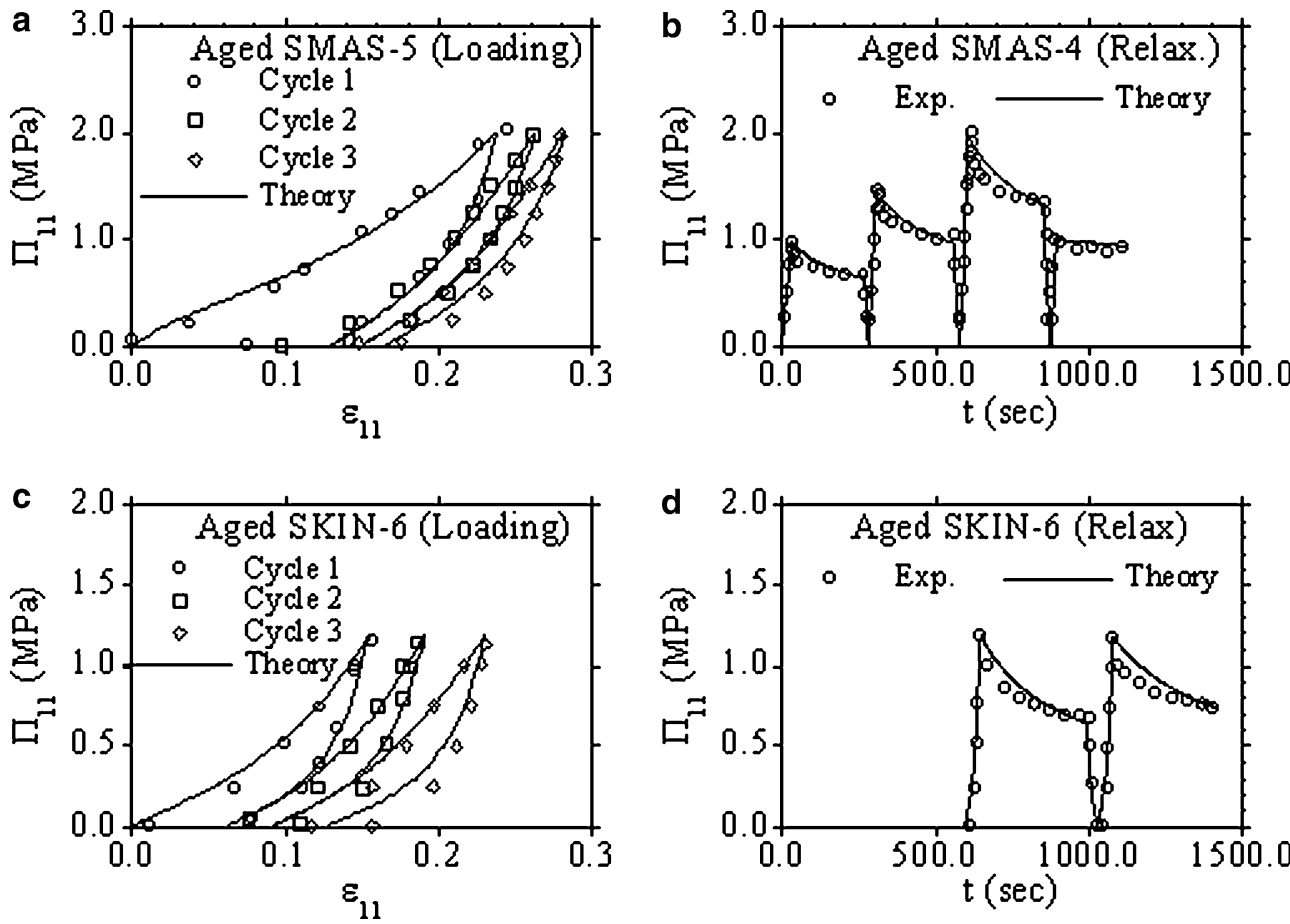
Fig. 4 Comparison of the theoretical results for aged tissues with those for young tissues for cyclic loading and relaxation tests. First PiolaKirchhoff (engineering) stress $\Pi_{11}$ versus engineering strain $\varepsilon_{11}$
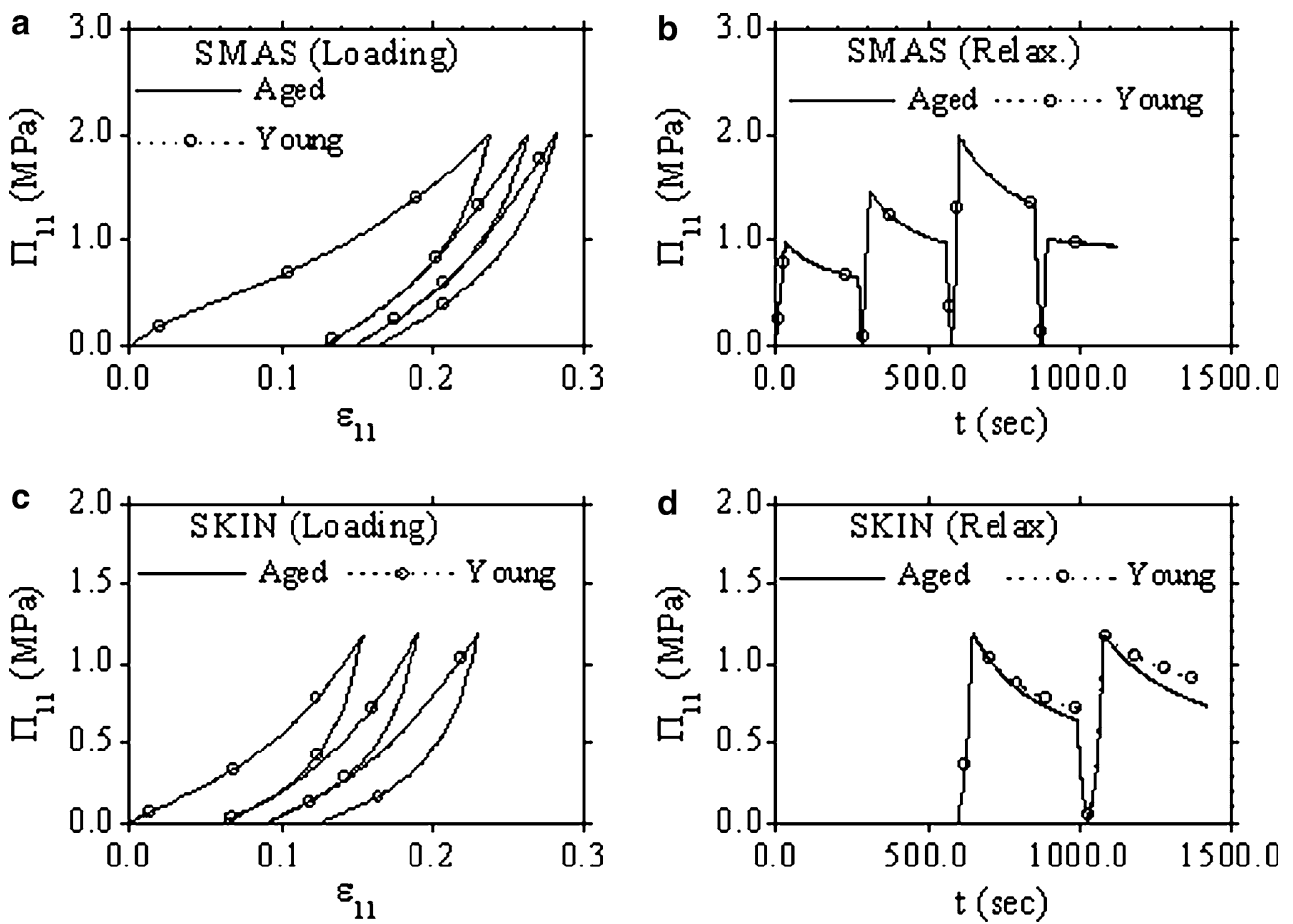

\section{Numerical modeling of the face}

A three-dimensional finite element model of a face was created using a triangulated surface obtained from a range laser scan (Vannier et al. 1991) of a young man. A multilayered structure made of three-dimensional elements was shaped according to the geometry of the triangulated surface (see Fig. 5) in order to model the layers of soft tissues of the anterior part of the head. Figure 5 also indicates the fixation points of the SMAS and deep fat layers using bold dots at the nodes of the element mesh. The skin and mucosa were not fixed at these nodes. Moreover, the nodes of all tissues at the model boundaries of the head and neck were fixed. These fixation regions correspond to the foundations described by Barton (2001) and LaTrenta (2004). Zygomatic ligaments, buccal-maxillary and mandibular ligaments, as well as platysma-auricolar ligaments support the facial tissues and counteract gravitational loads.

As mentioned previously, the facial tissue is modeled by four layers shown in Fig. 2. The four tissue layers are attached at their respective interfaces with no slip. The overall soft tissue thickness, which is approximately

Table 4 Strain rates for the experimental data

\begin{tabular}{lll}
\hline & $\begin{array}{l}\text { SMAS and } \\
\text { superficial fat } \dot{\varepsilon}_{11}\left(s^{-1}\right)\end{array}$ & Skin $\dot{\varepsilon}_{11}\left(s^{-1}\right)$ \\
\hline Cycle 1 & $\pm 2.0 \mathrm{E}-2$ & $\pm 1.0 \mathrm{E}-2$ \\
Cycle 2 & $\pm 5.0 \mathrm{E}-3$ & $\pm 2.5 \mathrm{E}-3$ \\
Cycle 3 & $\pm 1.0 \mathrm{E}-3$ & $\pm 5.0 \mathrm{E}-4$ \\
Loading and unloading & $\pm 5.0 \mathrm{E}-3$ & $\pm 2.5 \mathrm{E}-3$ \\
in relaxation cycles & & \\
\hline
\end{tabular}

constant over the present face model, does not reflect the variation in facial tissue thickness known for different face locations. This simplification has been adopted here in order to facilitate the creation of a three dimensional multilayered FEM (finite element model) model. Morphological data from medical imaging techniques could be used in future simulations in order to improve the realism of the geometrical model in terms of the thickness of the overall tissue composite as well as the thickness of each layer.

As noted in the literature (Rubin et al. 1998; Saulis et al. 2002), the SMAS acts as the firmer elastic foundation of the more viscous skin. One of the observations in the classical paper of Mitz and Peyronie (1976) is that superficial to the SMAS are fat lobules interwoven with fibrous septa which connect the SMAS with the dermis. A recent study of the elastic behavior of filament bundles appears in Gardel et al. (2004). The effective behavior of the SMAS therefore includes that of the filaments held in place by the fat lobules. In this regard, a simplistic mechanical interpretation of the facial structure is that the relatively coarse facial muscles activate the SMAS which transfers the motion to the smooth skin by the septa filaments held in place by the viscous fat lobules. In particular, the composite of fat lobules, septa and dermis tend to mask ripples due to muscle contractions leading to smooth facial expressions.

These constitutive equations have been implemented into the FEM program ABAQUS 6.3 (Hibbit et al. 2002). Specifically, special attention was focused on implementing the equations so that (i) they would be incrementally invariant under superposed rigid body motions, and (ii) the algorithm for integration of the evolution equation of the internal variables would con- 
Fig. 5 Initial and final meshes for 30 years of gravity loading. Also, the points associated with fixation ligaments are indicated on the initial mesh

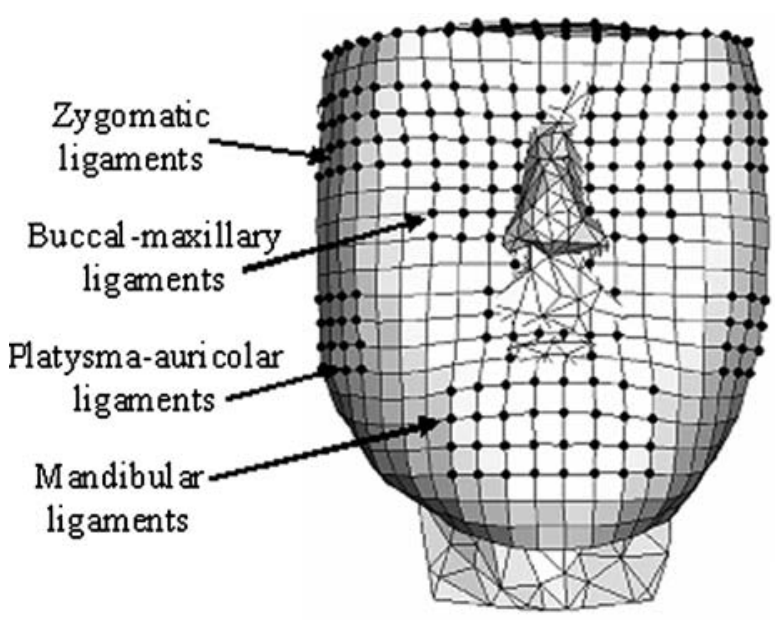

Initial

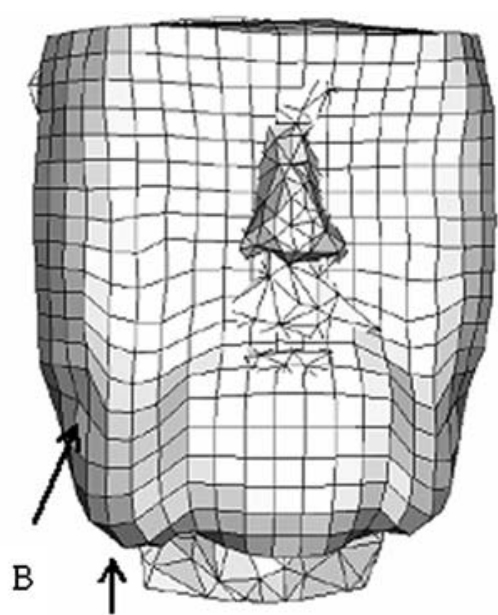

A verge without imposing severe restrictions on time stepping (Papes 2004). This FEM program is used for all of the present simulations of the face. The numerical mesh consists of 3840 nodes corresponding to 11520 degrees of freedom.

\section{Results}

Calculations were performed for 30 years of gravity loading and the initial and final meshes for these calculations are shown in Fig. 5. Extensive gravimetric soft tissue descent can be observed in the cheek and jowl regions. Figure 6 shows the vertical displacement of the point $\mathrm{A}$ indicated in Fig. 5 as a function of time. In particular, it can be seen that after the initial rapid drop caused by sudden application of the gravitational load, point A remains stable until the reduction of elastic stiffness due to aging becomes significant.

Figure 7 shows distributions of the maximum principal Cauchy stress in both the skin and SMAS layers at early (shortly after the tissues equilibrate the gravity load) and final stages of the calculation. It can be observed that in the final state the magnitude and extent of the high-stress regions in the SMAS layer are larger than those in the skin layer. This tends to confirm that the SMAS acts as the firmer elastic foundation of the more viscous skin (Rubin et al. 1998; Saulis et al. 2002). Figure 8 shows the final distribution of the aging parameter $\alpha$ in the skin and SMAS layers. Comparison of Figs. 7 and 8 shows that the stresses and the aging parameter $\alpha$ are highest near the fixation points of the platysma-auricolar ligaments (see Fig. 5) which support the tissue. Moreover, it is observed that the jowls droop around the fixation points of the mandibular ligaments.

Figure 9 shows the time history of the hardening parameter $\beta$ at the point B indicated in Fig. 5 in the skin layer. The sudden application of the gravitational load causes $\beta$ to increase due to stretching of the skin. Then, recovery of $\beta$ occurs as it returns to near zero value. The period (about $1-10$ years) in which $\beta$ remains near zero corresponds to the period shown in Fig. 6 where gravimetric descent is minimal and the region (about $0-0.4$ ) of $\alpha$ in Fig. 1 where the aging process is nearly inactive. For the period (about 10-30 years) the value of $\beta$ again increases and subsequently decreases due to recovery. This corresponds to the region (about $0.4-0.8$ ) of $\alpha$ in Fig. 1 where most of the aging occurs. It also corresponds to the period shown in Fig. 6 where most of the gravimetric descent occurs. This response of the hardening parameter $\beta$ is determined in the model by the constants $\left\{r_{1}, r_{2}, r_{3}, r_{4}, r_{5}\right\}$. In particular, the value of $r_{3}$ in Table 1 adopted here was specified in the previous work (Rubin and Bodner 2002) primarily to be small enough to separate the response to short-time cyclic loadings (with finite $\dot{\varepsilon}$ ) from that of relaxation tests (with vanishing $\dot{\varepsilon}$ ). In that work the effect of aging over a long time period of 30 years was not considered. Therefore, one direction of future experimental work could attempt to quantify the interactions of aging and hardening

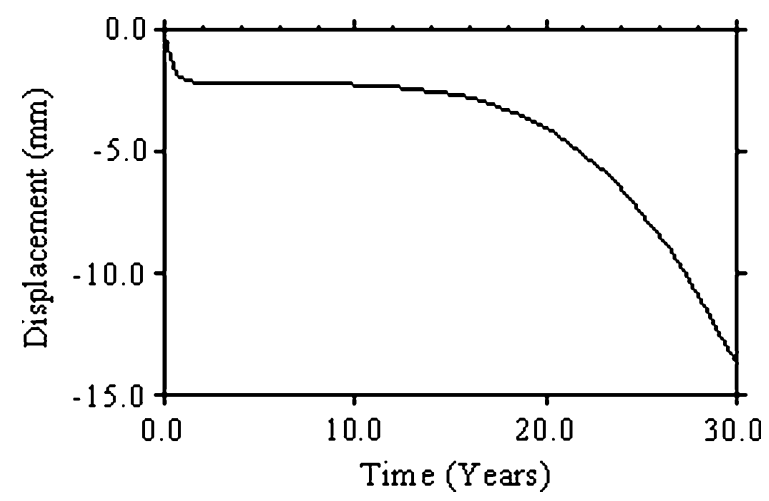

Fig. 6 Vertical displacement of the point A indicated in Fig. 5 as a function of time 
Fig. 7 Early and final distributions of the maximum principal Cauchy stress in both the skin and SMAS layers
Early

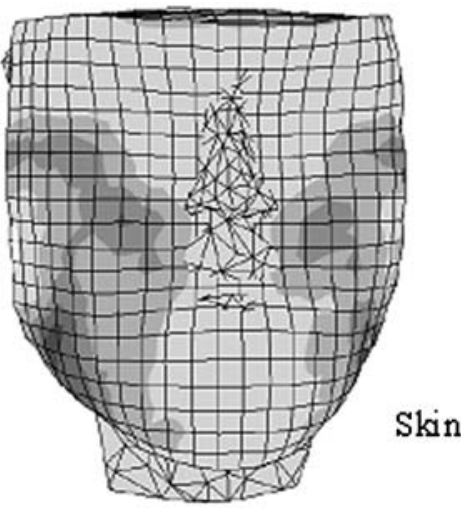
$+8.125 e-05$ -5.000 e-05
Final
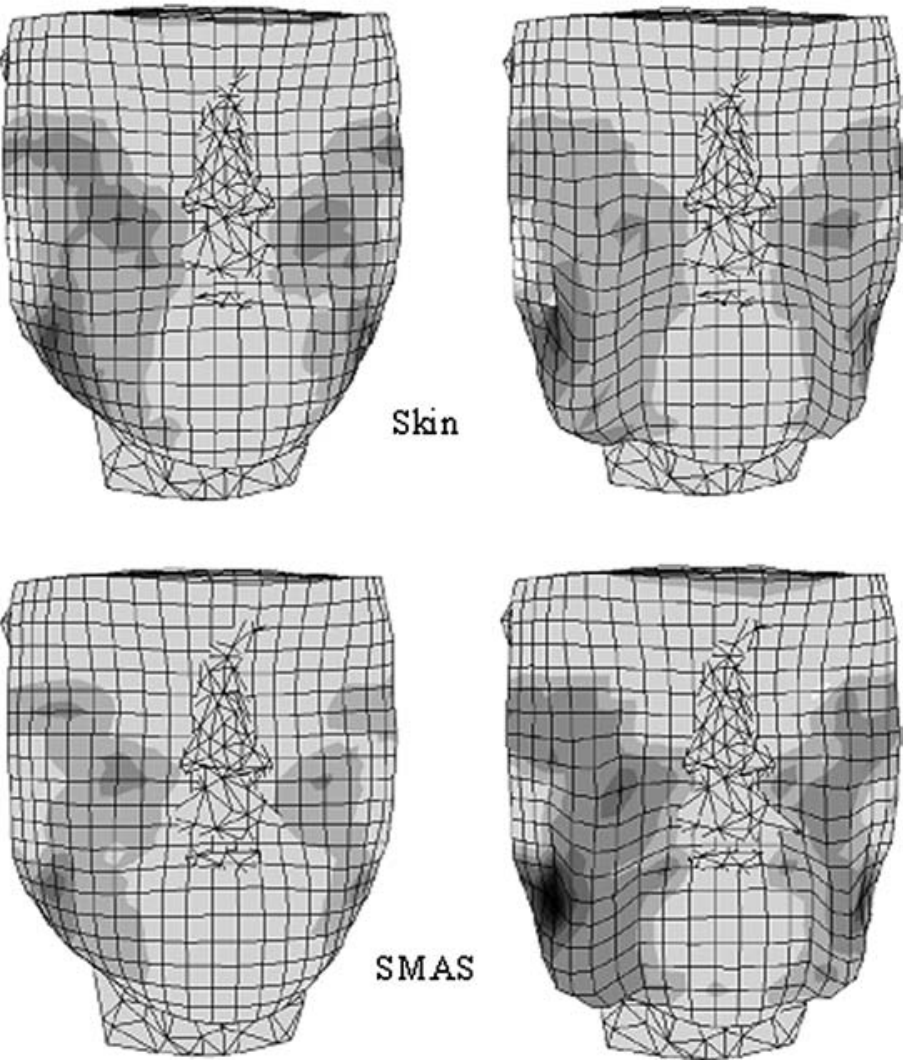

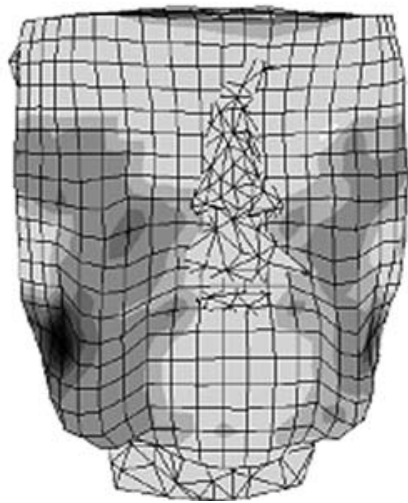

Fig. 8 Final distributions of the aging parameter $\alpha$ in the skin and SMAS layers
Skin

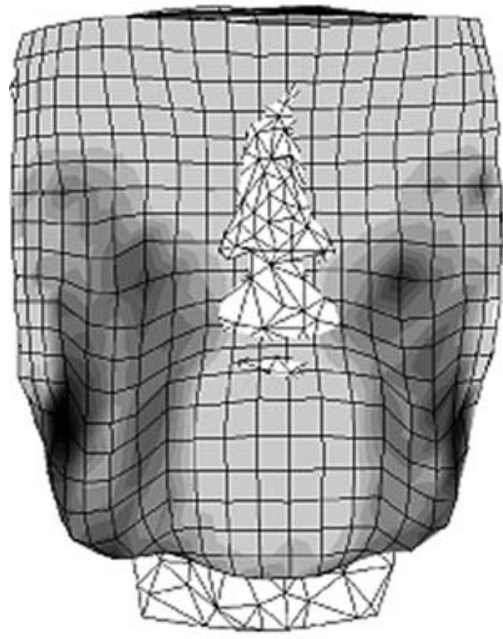

SMAS

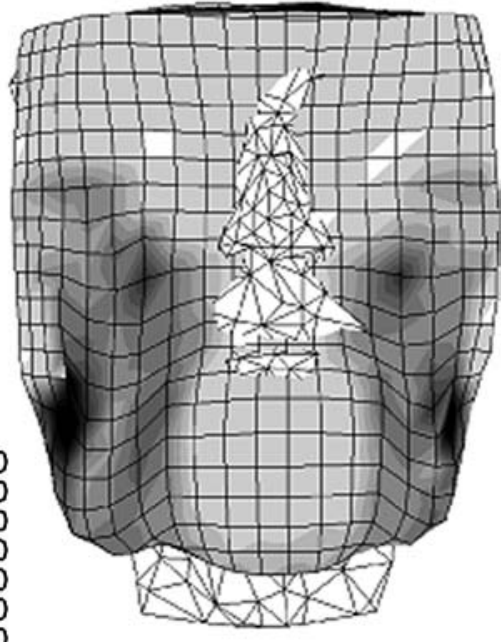

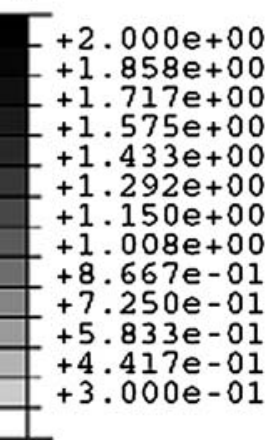




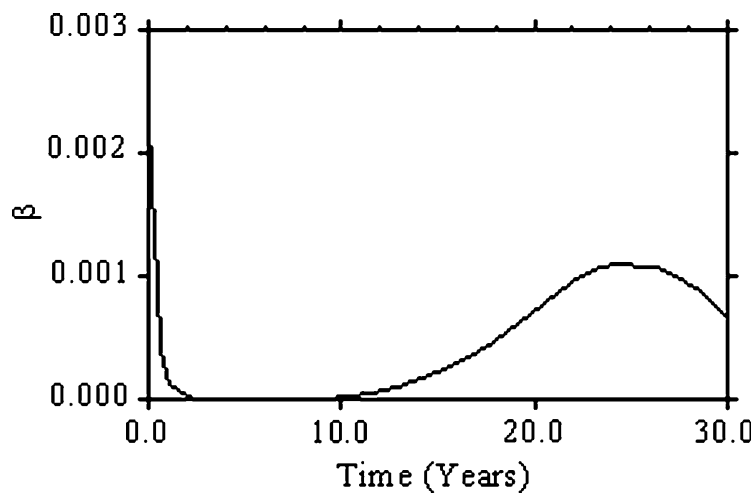

Fig. 9 Time history of the hardening parameter $\beta$ at the point $\mathrm{B}$ indicated in Fig. 5 in the skin layer

during long-time response which would lead to appropriate modifications of the evolution Eq. 20, the functional form Eq. 21, and the evolution Eq. 24.

\section{Conclusions and future research}

It is obvious, that the model proposed in this work cannot reproduce all the complex phenomena that characterize the aging process of a human face. Nevertheless, the present results demonstrate the feasibility of threedimensional mechanical analyses of the face with a FEM computer code utilizing constitutive relations which reflect the highly nonlinear elastic-viscoplastic behavior of facial soft tissues. In this work, emphasis has been placed on the physical realism of the mechanical response of the tissue. In particular, most of the important material constants in the constitutive equations have been determined to match short-time cyclic loading and relaxation tests (Har-Shai et al. 1996, 1997; Rubin et al. 1998; Rubin and Bodner 2002). It has been shown that this short-time response is dominated by the dissipative viscoplastic response of the tissue, whereas the long-time response to gravity loading is controlled by the stiffness of the aging elastic components in the tissues. For the derived material constants, the short-time response of both young and aged tissues are nearly identical. Therefore, the representation of both elastic and viscoplastic behavior is required for the computational model to be useful for practical purposes, such as providing a quantitative assessment of alternative surgical procedures.

Three main features characterize the present threedimensional face analysis with respect to previous models for face simulations: (i) the facial tissue is modeled as a multilayered structure, (ii) the short-time mechanical behavior of the SMAS and skin tissue layers is based on experimental data from mechanical tests on excised tissue samples, and (iii) the procedure developed for treating aging effects leads to consistent and reasonable predictions. These characteristics support the use of the present approach for possible future research, such as:
1. Additional studies of the role of the different tissue layers in the mechanical behavior of the face. The results of the present calculation highlighted the role of SMAS as the supportive tissue.

2. Further investigations of face aging mechanisms, for example, the dependence on the histories of straining and physiological and environmental effects.

3. The study of the influence of time dependent load induced extension of the retaining ligaments (relaxation of the fixation points) and possible remodeling of the tissues on the long time aging process.

4. Comparison of different procedures for cosmetic surgery with respect to short time results and longevity. Layer separation and stretching procedures as well as suturing techniques can be simulated with three dimensional or simplified two dimensional FEM models.

5. Investigation of the role of detailed anatomical data for fixation ligaments on modeling the results of different surgical procedures.

6. Use of active contractile elements in the FEM model to simulate the effects of muscles on facial expressions.

7. Investigation of the wrinkling in the forehead region and near the eyes caused by muscle lengthening and loss of muscle tone (LaTrenta, 2004).

Acknowledgements The authors thank Dr. Matthias Teschner of the Computer Graphics Laboratory of ETH Zurich for providing the triangulated face surface and for his precious advice on the creation of the three-dimensional element mesh. Also, the work of M.B. Rubin was partially supported by the fund for promotion of research at the Technion. In addition, the authors wish to thank the reviewers for their constructive comments.

\section{References}

Avis NJ (2000) Virtual environment technologies. J Minimally Invasive Therapy Allied Technol 9:333-339

Barton FE Jr (2001) Review article: the aging face, rhytidectomy and adjunctive procedures. Selected Readings in Plastic Surgery. 9(19), Baylor University Medical Center, Dallas

Bodner SR, Chan KS (1986) Modeling of continuum damage for application in elastic-viscoplastic constitutive equations. Eng Fract Mech 25:705-712

Bodner SR, Partom Y (1975) Constitutive equations for elasticviscoplastic strain-hardening materials. J Appl Mech 42:385389

Brett PN, Fraser CA, Henningam M, Griffiths MV, Kamel Y (1995) Automatic surgical tools for penetrating flexible tissues. IEEE Eng Med Biol 14:264-270

Burdea G (1996) Force and touch feedback for virtual reality. Wiley, New York

Contet-Audonneau JL, Jeanmaire C, Pauly G (1999) A histological study of human wrinkle structures: comparison between sunexposed areas of the face, with or without wrinkles, and sunprotected areas. Br J Dermatol 140:1038-1047

Cotta-Pereira G, Guerro-Rodriguez F, Bittencourt-Sampaio S (1978) Oxytalan, elaunin and the elastic fibres in the human skin. J Investig Dermatol 66:143-148 
Craven NM, Watson REB, Jones CJP, Shuttleworth CA, Kielty CM, Griffiths CEM (1997) Clinical features of photoaged human skin are associated with a reduction in collagen VI. Br J Dermatol 137:344-350

Donofrio LM (2000) Fat distribution: a morphologic study of the aging face. Dermatol Surg 26:1107-1112

Fleischmajer R, Perlish JS, Bashey RI (1972) Human dermal glycosaminoglycans and aging. Biochim Biophys Acta 279:265-275

Flory P (1961) Thermodynamic relations for high elastic materials. Trans Faraday Soc 57:829-838

Fung YC (1993) Biomechanics, Mechanical Properties of Living Tissues, 2nd edn. Springer-Verlag, Berlin Heidelberg New York

Gardel ML, Shin JH, MacKintosh FC, Mahadevan L, Matsudaira P, Weitz DA (2004) Elastic behavior of cross-linked and bundled actin networks. Science 304:1301-1305

Har-Shai Y, Bodner SR, Egozy-Golan D, Lindenbaum ES, BenIzhak O, Mitz V, Hirshowitz B (1996) Mechanical properties and microstructure of the superficial musculoaponeurotic system. Plast Reconstr Surg 98:59-70

Har-Shai Y, Bodner SR, Egozy-Golan D, Lindenbaum ES, BenIzhak O, Mitz V, Hirshowitz B (1997) Viscoelastic properties of the superficial musculoaponeurotic system: a microscopic and mechanical study. Aesth Plast Surg 21:219-224

Har-Shai Y, Sela E, Rubinstien I, Lindenbaum ES, Mitz V, Hirshowitz B (1998) Computerized morphometric quantitation of elastin and collagen in SMAS and facial skin and the possible role of fat cells in SMAS viscoelastic properties. Plast Reconstr Surg 102:2466-2470

Hibbit HD, Karlsson BI,Sorensen P (2002) ABAQUS theory manual. Version 6.3 .

Koch RM, Gross MH, Carls FR, von Büren DF, Fankhauser G, Parish YIH (1996) Simulating facial surgery using finite element models. Proc SIGGRAPH' 96:421-427
LaTrenta G (2004) Aesthetic face \& neck surgery. Saunders, Philadelphia

Lee Y, Terzopoulis D, Waters K (1995) Realistic modeling for facial animations. Proc SIGGRAPH' 95:55-62

Mitz V, Peyronie M (1976) The superficial musculoaponeurotic system (SMAS) in the parotoid and cheek area. Plast Reconstr Surg 58:80-88

Papes O (2004) Part of a Ph.D. thesis in progress under the supervision of E. Mazza at ETH, Zurich

Parke FI (1982) Parameterized models for facial animation. IEEE Comput Graphics Appl 2:61-68

Rubin MB (1994) Plasticity theory formulated in terms of physically based microstructural variables - Part I: theory. Int J Solids Structures 31:2615-2634

Rubin MB, Bodner SR (2002) A three-dimensional nonlinear model for dissipative response of soft tissue. Int $\mathbf{J}$ Solids Structures 39:5081-5099

Rubin MB, Bodner SR, Binur NS (1998) An elastic-viscoplastic model for excised facial tissue. J Biomech Eng 120:686-689

Saulis AS, Lautenschlager EP, Mustoe TA (2002) Viscoelastic properties of skin, SMAS, and composite flaps as they pertain to rhytidectomy. Plast Reconstr Surg 110:590-598

Snedeker JG, Bajka M, Hug JM, Szekely G, Niederer P (2002) The creation of a high-fidelity finite element model of the kidney for use in trauma research. $\mathbf{J}$ Visualization Compu Animation 13:53-64

Szekely G (2003) Surgical simulators. Minimally Invasive Therapy Allied Technol 12:14-18

Vannier MW, Pilgram T, Bhatia G, Brunsden B, Commean P (1991) Facial surface scanner. IEEE Comput Graph Appl 11:72-80

Zhang Y, Prakash EC, Sung E (2004) Face alive. J Visual Languages Computing 15:125-160 